\title{
A quantitative version of Krein's Theorem
}

\section{Fabian, P. Hájek, V. Montesinos and V. Zizler}

To the memory of Vlastimil Pták

\begin{abstract}
A quantitative version of Krein's Theorem on convex hulls of weak compact sets is proved. Some applications to weakly compactly generated Banach spaces are given.
\end{abstract}

\section{Introduction}

A natural question related to the classical Theorem of Krein (see, for example, [6, Theorem 3.58]) is the following: assume that a bounded subset $M$ of a Banach space $X$ satisfies

$$
\bar{M}^{w^{*}} \subset X+\varepsilon B_{X^{* *}}
$$

for some $\varepsilon \geq 0$. Does the same hold for its convex hull? (if $\varepsilon=0$ the answer is "yes" and this is the statement of Krein's Theorem). To answer in the affirmative this apparently simple question turns to be quite elusive in general.

This question arose when studying the problem of characterizing subspaces of weakly compact generated Banach spaces by countable covers of its closed unit ball (see [5] and Theorem 15 in section 3 below).

The following definition describes the central object in this note.

Definition 1 Let $X$ be a Banach space and let $M$ be a bounded subset of $X$. Given $\varepsilon \geq 0$, we say that $M$ is $\varepsilon$-weakly relatively compact $(\varepsilon-W R K$, for short) if

$$
\bar{M}^{w^{*}} \subset X+\varepsilon B_{X^{* *}} .
$$

The case $\varepsilon=0$ is the classical weakly relatively compactness.

2000 Mathematics Subject Classification: 46A50, 46B50.

Keywords: Banach spaces, weak compactness, Krein's Theorem. 
Using techniques of double limits due to Grothendieck and Pták, we will prove that the answer to the former question for any $\varepsilon \geq 0$ is affirmative for Banach spaces $X$ with $\omega^{*}$-angelic dual closed unit ball ${ }^{1}$ (in particular, separable Banach spaces - a result due to Rosenthal, see the acknowledgements at the end of this note -or, more generally, weakly compactly generated or even weakly Lindelöf determined Banach spaces). Moreover, if a relaxation to $2 \varepsilon$ of the constant is allowed, it holds true for any Banach space. The following is the main result of this note.

Theorem 2 Let $(X,\|\cdot\|)$ be a Banach space. Let $M \subset X$ be a bounded subset of $X$. Assume that $M$ is $\varepsilon-W R K$ for some $\varepsilon>0$. Then conv $(M)$ is $2 \varepsilon-W R K$. If $\left(B_{X^{*}}, \omega^{*}\right)$ is angelic, or if $X^{*}$ does not contain a copy of $\ell^{1}$, then $\operatorname{conv}(M)$ is $\varepsilon-W R K$.

The following, to our knowledge, is still open²

Problem 3 Let $X$ be a Banach space. Let $M$ be a $\varepsilon$-weakly relatively compact subset of $X$. Is conv $(M) \varepsilon$-weakly relatively compact?

Remark 4 The decisive case for Problem 3 seems to be the space $\ell^{\infty}$. The answer should be related to the so-called boundary problem (see [2, Problem I.2]): Let $X$ be a Banach space and $B$ a subset of $S_{X^{*}}$ such that every $x \in X$ attains its norm at some point of $B$ ( $B$ is called a boundary of $X$ ). Let $A$ be a bounded subset of $X$ that is compact for the topology of the pointwise convergence on $B$. Is A weakly compact? (see [2, Chap. I] and the references therein).

\section{Proofs}

Given a Banach space $X$ and an element $x^{* *} \in X^{* *}$, the following function on $\left(B_{X^{*}}, w^{*}\right)$ is introduced in [2, III.2, p. 105]:

$$
\hat{x}^{* *}: B_{X^{*}} \rightarrow \mathbb{R}
$$

is the infimum of the real continuous functions on $\left(B_{X^{*}}, w^{*}\right)$ which are greater than or equal to $x^{* *}$. The following proposition gives two alternative descriptions of $\hat{x}^{* *}$. The first one is a standard result in general topology. The second one is in [2, III.2.3].

\footnotetext{
${ }^{1} \mathrm{~A}$ topological space $T$ is called angelic if every relatively countably compact set $A \subset T$ is relatively compact and if every point in $\bar{A}$ is the limit of a sequence in $A$.

${ }^{2}$ See the remark added in proof at the end of this paper.
} 
Proposition 5 Let $X$ be a Banach space. Then, given $x^{* *} \in X^{* *}$, (i)

$$
\hat{x}^{* *}\left(x_{0}^{*}\right)=\lim _{N \in \mathcal{N}\left(x_{0}^{*}\right)}\left\{\sup \left\langle x^{* *}, N\right\rangle\right\}, \quad \forall x_{0}^{*} \in B_{X^{*}},
$$

where $\mathcal{N}\left(x_{0}^{*}\right)$ denotes the filter of neighborhoods of $x_{0}^{*}$ in $\left(B_{X^{*}}, w^{*}\right)$.

(ii)

$$
\hat{x}^{* *}\left(x_{0}^{*}\right)=\inf \left\{\left\langle x, x_{0}^{*}\right\rangle+\left\|x^{* *}-x\right\| ; x \in X\right\}, \forall x_{0}^{*} \in B_{X^{*}} .
$$

Remark 6 In particular, it follows from $(i i)$ that if $d:=\operatorname{dist}\left(x^{* *}, X\right)$ denotes the distance in the norm from $x^{* *}$ to $X$ then $\hat{x}^{* *}(0)=d$. From $(i)$ we get then that for every $N \in \mathcal{N}(0), d \leq \sup \left\langle x^{* *}, N\right\rangle$, and for every $\varepsilon>0$, there exists $N_{\varepsilon} \in \mathcal{N}(0)$ such that $\sup \left\langle x^{* *}, N_{\varepsilon}\right\rangle<d+\varepsilon$.

The use of double limits in the study of compactness is implicit in the approach of Eberlein [4] and explicit in Grothendieck (see, for example, [10]). The following concept relaxes the usual double limit condition.

Definition 7 Let $M$ be a bounded set of a Banach space $X$, and let $S$ be a bounded subset of $X^{*}$. We say that $M \varepsilon$-interchanges limits with $S$ (and in this case we shall write $M \S \varepsilon \oint S)$ if for any two sequences $\left(x_{n}\right)$ in $M$ and $\left(x_{m}^{*}\right)$ in $S$ such that the following limits exist,

$$
\lim _{n} \lim _{m}\left\langle x_{n}, x_{m}^{*}\right\rangle, \quad \lim _{m} \lim _{n}\left\langle x_{n}, x_{m}^{*}\right\rangle,
$$

then

$$
\left|\lim _{n} \lim _{m}\left\langle x_{n}, x_{m}^{*}\right\rangle-\lim _{m} \lim _{n}\left\langle x_{n}, x_{m}^{*}\right\rangle\right| \leq \varepsilon .
$$

Proposition 8 Let $M$ be a bounded set and $\varepsilon \geq 0$ some number. Then we have

(i) If $M$ is $\varepsilon-W R K$ then $M \S 2 \varepsilon \oint B_{X^{*}}$.

(ii) If $M \S \varepsilon \S B_{X^{*}}$ then $M$ is $\varepsilon-W R K$.

Proof. $(i)$ Let $\left(x_{n}\right)$ and $\left(x_{m}^{*}\right)$ be sequences in $M$ and $B_{X^{*}}$, respectively, such that both limits

$$
\lim _{n} \lim _{m}\left\langle x_{n}, x_{m}^{*}\right\rangle, \quad \lim _{m} \lim _{n}\left\langle x_{n}, x_{m}^{*}\right\rangle
$$

exist. Let $x^{* *} \in \bar{M}^{w^{*}}$ be a $w^{*}-$ cluster point of $\left(x_{n}\right)$. Then

$$
\lim _{n}\left\langle x_{n}, x_{m}^{*}\right\rangle=\left\langle x^{* *}, x_{m}^{*}\right\rangle, \forall m .
$$


Fix $\delta>0$. We can find $x \in X$ such that $\left\|x^{* *}-x\right\| \leq \varepsilon+\delta$. Choose a subsequence of $\left(x_{m}^{*}\right)$ (denoted again by $\left(x_{m}^{*}\right)$ ) such that $\lim _{m}\left\langle x, x_{m}^{*}\right\rangle$ exists. Let $x^{*} \in X^{*}$ be a $w^{*}$-cluster point of $\left(x_{m}^{*}\right)$. We get

$$
\begin{aligned}
\lim _{m}\left\langle x_{n}, x_{m}^{*}\right\rangle & =\left\langle x_{n}, x^{*}\right\rangle, \forall n, \\
\lim _{n} \lim _{m}\left\langle x_{n}, x_{m}^{*}\right\rangle & =\lim _{n}\left\langle x_{n}, x^{*}\right\rangle=\left\langle x^{* *}, x^{*}\right\rangle,
\end{aligned}
$$

and then

$$
\begin{gathered}
\left|\lim _{n} \lim _{m}\left\langle x_{n}, x_{m}^{*}\right\rangle-\lim _{m} \lim _{n}\left\langle x_{n}, x_{m}^{*}\right\rangle\right|=\left|\lim _{n}\left\langle x_{n}, x^{*}\right\rangle-\lim _{m}\left\langle x^{* *}, x_{m}^{*}\right\rangle\right|= \\
=\left|\left\langle x^{* *}, x^{*}\right\rangle-\lim _{m}\left\langle x^{* *}, x_{m}^{*}\right\rangle\right|=\left|\lim _{m}\left\langle x^{* *}, x^{*}-x_{m}^{*}\right\rangle\right| \leq \\
\leq\left|\lim _{m}\left\langle x, x^{*}-x_{m}^{*}\right\rangle\right|+2(\varepsilon+\delta)=2(\varepsilon+\delta) .
\end{gathered}
$$

As $\delta>0$ is arbitrary, we get the conclusion.

(ii) Assume now $M \S \varepsilon \S B_{X^{*}}$. Let $x^{* *} \in \bar{M}^{w^{*}}$ and let $d:=d\left(x^{* *}, X\right)$. We shall define inductively two sequences, $\left(x_{n}\right)$ in $M$ and $\left(x_{m}^{*}\right)$ in $B_{X^{*}}$. To begin with, choose any $x_{1} \in M$. Define then $N\left(x_{1} ; 1\right):=\left\{x^{*} \in B_{X^{*}} ;\left|\left\langle x_{1}, x^{*}\right\rangle\right|<1\right\}$, a neighbourhood of 0 in $\left(B_{X^{*}}, w^{*}\right)$. By Remark 6 we can find $x_{1}^{*} \in N\left(x_{1} ; 1\right)$ such that

$$
d-1 \leq\left\langle x^{* *}, x_{1}^{*}\right\rangle<d+1 .
$$

Choose $x_{2} \in M$ such that $\left|\left\langle x^{* *}-x_{2}, x_{1}^{*}\right\rangle\right|<1 / 2$. Define $N\left(x_{1}, x_{2} ; 1 / 2\right):=$ $\left\{x^{*} \in B_{X^{*}} ;\left|\left\langle x_{i}, x^{*}\right\rangle\right|<1 / 2, i=1,2\right\}$, a neighborhood of 0 in $\left(B_{X^{*}}, w^{*}\right)$. Again by Remark 6 we can find $x_{2}^{*} \in N\left(x_{1}, x_{2} ; 1 / 2\right)$ such that $d-1 / 2 \leq$ $\left\langle x^{* *}, x_{2}^{*}\right\rangle<d+1 / 2$. Continue in this way. We get $\left(x_{n}\right)$ and $\left(x_{m}^{*}\right)$ such that

$$
\begin{aligned}
& x_{n} \in M, x_{m}^{*} \in B_{X^{*}}, \forall n, m, \\
&\left|\left\langle x^{* *}-x_{n}, x_{m}^{*}\right\rangle\right|<\frac{1}{n}, \quad m=1,2, \ldots, n-1, \\
&\left|\left\langle x_{n}, x_{m}^{*}\right\rangle\right|<\frac{1}{m}, \quad n=1,2, \ldots, m, \\
& d-\frac{1}{m} \leq\left\langle x^{* *}, x_{m}^{*}\right\rangle<d+\frac{1}{m}, \quad m=1,2, \ldots
\end{aligned}
$$

Then

$$
\begin{aligned}
\lim _{n}\left\langle x_{n}, x_{m}^{*}\right\rangle & =\left\langle x^{* *}, x_{m}^{*}\right\rangle, \forall m, \\
\lim _{m} \lim _{n}\left\langle x_{n}, x_{m}^{*}\right\rangle & =\lim _{m}\left\langle x^{* *}, x_{m}^{*}\right\rangle=d, \\
\lim _{m}\left\langle x_{n}, x_{m}^{*}\right\rangle & =0, \forall n, \\
\lim _{n} \lim _{m}\left\langle x_{n}, x_{m}^{*}\right\rangle & =0,
\end{aligned}
$$

so

$$
\left|\lim _{m} \lim _{n}\left\langle x_{n}, x_{m}^{*}\right\rangle-\lim _{n} \lim _{m}\left\langle x_{n}, x_{m}^{*}\right\rangle\right|=d \leq \varepsilon
$$


Remark 9 The case $\varepsilon=0$ gives Grothendieck's characterization of relatively weak compactness (see [10]).

Remark 10 In Proposition 8, $(i)$ cannot be improved. There are examples where $B_{X}$ is (obviously) 1-WRK although $B_{X} \oint \varepsilon \oint B_{X^{*}}$ is false for every $0<\varepsilon<2$. A simple instance is provided by $X:=\left(\ell^{1},\|\cdot\|_{1}\right)$ : let $\mathcal{N}$ be a non-trivial ultrafilter on $\mathbb{N}$ and, for every $u \in \ell^{\infty}$, let $\left\langle x^{* *}, u\right\rangle$ be the limit of $u$ along the ultrafilter $\mathcal{N}$. By using $(i)$ in Proposition 5 we get easily that $\left\langle x^{* *}, x^{*}\right\rangle=1$ for all $x^{*} \in B_{X^{*}}$, and this implies that, for every $0<\delta<1$, $S\left(x^{* *} ; \delta\right)$ is dense in $\left(B_{X^{*}}, \omega^{*}\right)$, where

$$
S\left(x^{* *} ; \delta\right):=\left\{x^{*} \in B_{X^{*}} ;\left\langle x^{* *}, x^{*}\right\rangle>1-\delta\right\}
$$

(see Proposition 11 below). Choose $0<\delta<(2-\varepsilon) / 2$ and an element $x^{*} \in S\left(-x^{* *} ; \delta\right)$. We can find then a sequence $\left(x_{m}^{*}\right)$ (as $\left(B_{X^{*}}, w^{*}\right)$ is metrizable) in $S\left(x^{* *} ; \delta\right)$ such that $x_{m}^{*} \rightarrow x^{*}$ in the $w^{*}$-topology. By a diagonal procedure we can choose a sequence $\left(x_{n}\right)$ in $B_{X}$ such that $x_{n} \rightarrow x^{* *}$ on the set $\left\{x^{*}, x_{m}^{*} ; m \in \mathbb{N}\right\}$. Then we have

$$
\begin{aligned}
\left|\lim _{n} \lim _{m}\left\langle x_{n}, x_{m}^{*}\right\rangle-\lim _{m} \lim _{n}\left\langle x_{n}, x_{m}^{*}\right\rangle\right| & =\left|\lim _{n}\left\langle x_{n}, x^{*}\right\rangle-\lim _{m}\left\langle x^{* *}, x_{m}^{*}\right\rangle\right| \\
& =\left|\left\langle x^{* *}, x^{*}\right\rangle-\lim _{m}\left\langle x^{* *}, x_{m}^{*}\right\rangle\right|= \\
& =\left|\lim _{m}\left\langle x^{* *},\left(x^{*}-x_{m}^{*}\right)\right\rangle\right|>2-2 \delta>\varepsilon .
\end{aligned}
$$

and the assertion is proved.

The construction in the previous example can be carried over to every separable Banach space $X$ which contains an isomorphic copy of $\ell^{1}$. It follows that an equivalent norm can be found on $X$ such that, in this norm, $B_{X}$ is (obviously) 1 -WRK although $B_{X} \oint \varepsilon \oint B_{X^{*}}$ is false for every $0<\varepsilon<2$. The argument depends on the notion of an octahedral norm. A norm $\|\cdot\|$ on $X$ is said to be octahedral (see, for example, [2, III.2]) if for every finite dimensional subspace $F$ of $X$ and every $\eta>0$, there exists $y \in S_{X}$ such that for every $x \in F$, we have

$$
\|x+y\| \geq(1-\eta)(\|x\|+1) .
$$

By [2, Lemma III.2.2], if there exists $x^{* *} \in X^{* *} \backslash\{0\}$ such that

$$
\left\|x^{* *}+x\right\|=\left\|x^{* *}\right\|+\|x\| \quad \text { for every } x \in X,
$$

then $\|\cdot\|$ is octahedral. The converse implication is true if $X$ is separable ([7]). The following proposition characterizes such elements $x^{* *}$ in $X^{* *}$. 
Proposition 11 Let $X$ be a Banach space and let $x^{* *} \in S_{X^{* *}}$. The following assertions are equivalent:

(i) $\left\|x^{* *}+x\right\|=\left\|x^{* *}\right\|+\|x\|$ for every $x \in X$.

(ii) $\hat{x}^{* *}\left(x^{*}\right)=1$, for every $x^{*} \in B_{X^{*}}$.

(iii) For every $0<\delta<1, S\left(x^{* *} ; \delta\right)$ is dense in $\left(B_{X^{*}}, w^{*}\right)$, where

$$
S\left(x^{* *} ; \delta\right):=\left\{x^{*} \in B_{X^{*}} ;\left\langle x^{* *}, x^{*}\right\rangle>1-\delta\right\} .
$$

Proof. The equivalence between $(i)$ and $(i i)$ is proved in [2, III.2.4].

(ii) $\Rightarrow($ iii $)$. Let $x_{0}^{*} \in B_{X^{*}}$. Let $N_{1}\left(x_{0}^{*}\right)$ be a neighborhood of $x_{0}^{*}$ in $\left(B_{X^{*}}, w^{*}\right)$. By Proposition 5 , given $0<\delta<1$ we can find $N_{2}\left(x_{0}^{*}\right) \subset N_{1}\left(x_{0}^{*}\right)$, a neighborhood of $x_{0}^{*}$ in $\left(B_{X^{*}}, w^{*}\right)$, such that $\sup \left\langle x^{* *}, N_{2}\left(x_{0}^{*}\right)\right\rangle \geq 1$. Choose $x^{*} \in N_{2}\left(x_{0}^{*}\right)$ such that $\left\langle x^{* *}, x^{*}\right\rangle>1-\delta$. Then

$$
x^{*} \in S\left(x^{* *} ; \delta\right) \cap N_{1}\left(x_{0}^{*}\right) .
$$

It follows that $S\left(x^{* *} ; \delta\right)$ is dense in $\left(B_{X^{*}}, w^{*}\right)$.

$($ iii $) \Rightarrow($ ii $)$ follows from Proposition 5 .

Now, in any separable Banach space $X$ containing an isomorphic copy of $\ell^{1}$ there exists an octahedral equivalent norm $\|\cdot\| \|$, and according to [7], there exists $x^{* *} \in S_{X^{* *}}$ such that $\left\|x^{* *}+x\right\|\|=\| x^{* *}\|+\| x \|$ for every $x \in X$. The rest of the argument follows from Proposition 11 as in the example.

The proof of the following theorem is a quantitative modification of the proof of Krein's Theorem due to Pták, in which he used his combinatorial lemma together with Grothendieck's double limit criterion (see, for example, [12], [11, §24.5] or [1]).

We need the following definitions.

$$
C(\mathbb{N}):=\{\lambda: \mathbb{N} \rightarrow[0,1]: \text { supp } \lambda \text { finite }, \lambda(\mathbb{N})=1\},
$$

where supp $\lambda$ denotes the support of $\lambda$, i.e., the set $\{n \in \mathbb{N}: \lambda(n) \neq 0\}$, and $\lambda(B):=\sum_{n \in B} \lambda(n)$ for any $B \subset \mathbb{N}$. Let $\mathcal{G}$ be a family of finite subsets of $\mathbb{N}$. Given $B \subset \mathbb{N}$, let

$$
C(B):=\{\lambda \in C(\mathbb{N}): \text { supp } \lambda \subset B\} .
$$


Given $\gamma>0$, let

$$
C(B, \mathcal{G}, \gamma):=\{\lambda \in C(B): \lambda(G)<\gamma, \forall G \in \mathcal{G}\} .
$$

Pták's Combinatorial Lemma reads

Lemma 12 (Pták [12]) The two following conditions on $\mathcal{G}$ are equivalent:

1. There exists a strictly increasing sequence $A_{1} \subset A_{2} \subset \cdots$ of finite subsets of $\mathbb{N}$ and a sequence $\left(G_{n}\right)$ in $\mathcal{G}$ with $A_{n} \subset G_{n}$ for all $n$.

2. There exists an infinite subset $B \subset \mathbb{N}$ and an $\gamma>0$ such that

$$
C(B, \mathcal{G}, \gamma)=\emptyset
$$

Theorem 13 Let $(X,\|\cdot\|)$ be a Banach space. Let $M \subset X$ be a bounded subset of $X$. Assume that $M \S \varepsilon \S B_{X^{*}}$ for some $\varepsilon \geq 0$. Then

$$
\operatorname{conv}(M) \S \varepsilon \oint B_{X^{*}} \text {. }
$$

Proof. Assume $\|x\| \leq \mu$ for all $x \in M$ and some $\mu>0$. Choose $\varepsilon>0$ and $0<\beta<\varepsilon$. Select now $\delta>0$ and $\gamma>0$ such that $\beta+2 \gamma \mu<\varepsilon-\delta$. Suppose that there exists a sequence $\left(x_{n}\right)$ in $\operatorname{conv}(M)$ and a sequence $\left(x_{m}^{*}\right)$ in $B_{X^{*}}$ such that

$$
\left|\lim _{n} \lim _{m}\left\langle x_{n}, x_{m}^{*}\right\rangle-\lim _{m} \lim _{n}\left\langle x_{n}, x_{m}^{*}\right\rangle\right|=\varepsilon>0 .
$$

Let $x_{0}^{*} \in B_{X^{*}}$ be a cluster point of $\left(x_{m}^{*}\right)$ in $\left(B_{X^{*}}, w^{*}\right)$. Let $T \subset M$ be a countable set such that

$$
\left\{x_{n}: n \in \mathbb{N}\right\} \subset \operatorname{conv}(T)
$$

and choose a subsequence (denoted again by $\left(x_{m}^{*}\right)$ ) such that $x_{m}^{*} \rightarrow x_{0}^{*}$ on the set $T$. Then, for some $\sigma \in\{-1,1\}$,

$$
\sigma\left(\lim _{n}\left\langle x_{n}, x_{0}^{*}\right\rangle-\lim _{m} \lim _{n}\left\langle x_{n}, x_{m}^{*}\right\rangle\right)=\varepsilon .
$$

By suppressing a finite number of indices, we may assume

$$
\sigma\left(\lim _{n}\left\langle x_{n}, x_{0}^{*}\right\rangle-\lim _{n}\left\langle x_{n}, x_{m}^{*}\right\rangle\right)=\sigma \lim _{n}\left\langle x_{n}, x_{0}^{*}-x_{m}^{*}\right\rangle>\varepsilon-\delta, \forall m .
$$

Define

$$
\Gamma(t):=\left\{m \in \mathbb{N}:\left|\left\langle t, x_{0}^{*}-x_{m}^{*}\right\rangle\right| \geq \beta\right\}, t \in T .
$$

Those are finite subsets of $\mathbb{N}$. Let

$$
\mathcal{G}:=\{\Gamma(t): t \in T\} .
$$


Assume $C(\mathbb{N}, \mathcal{G}, \gamma) \neq \emptyset$ and choose $\lambda \in C(\mathbb{N}, \mathcal{G}, \gamma)$. It follows that

$$
\lambda(\Gamma(t))<\gamma, \forall t \in T .
$$

Form

$$
x^{*}:=\sum_{k \in \mathbb{N}} \lambda(k)\left(x_{0}^{*}-x_{k}^{*}\right) \in 2 B_{X^{*}}
$$

Given $t \in T$,

$$
\begin{aligned}
\left|\left\langle t, x^{*}\right\rangle\right| & =\left|\sum_{k \in \mathbb{N}} \lambda(k)\left\langle t, x_{0}^{*}-x_{k}^{*}\right\rangle\right| \leq \\
& \leq \sum_{\Gamma(t)} \lambda(k)\left|\left\langle t, x_{0}^{*}-x_{k}^{*}\right\rangle\right|+\sum_{\mathbb{N} \backslash \Gamma(t)} \lambda(k)\left|\left\langle t, x_{0}^{*}-x_{k}^{*}\right\rangle\right|<2 \gamma \mu+\beta .
\end{aligned}
$$

It follows that

$$
\left|\left\langle x_{n}, x^{*}\right\rangle\right| \leq 2 \gamma \mu+\beta, \forall n
$$

Then

$$
\begin{aligned}
2 \gamma \mu+\beta & \geq \lim _{n}\left|\left\langle x_{n}, x^{*}\right\rangle\right|= \\
& =\left|\sum_{k \in \mathbb{N}} \lambda(k) \lim _{n}\left\langle x_{n}, x_{0}^{*}-x_{k}^{*}\right\rangle\right|=\sigma \sum_{k \in \mathbb{N}} \lambda(k) \lim _{n}\left\langle x_{n}, x_{0}^{*}-x_{k}^{*}\right\rangle>\varepsilon-\delta,
\end{aligned}
$$

a contradiction.

Assume then $C(\mathbb{N}, \mathcal{G}, \gamma)=\emptyset$. Then, by Lemma 12 we can find $A_{p}:=$ $\left\{m_{1}, m_{2}, \ldots, m_{p}\right\} \subset \mathbb{N}$ and $t_{p} \in T$ such that

$$
A_{p} \subset \Gamma\left(t_{p}\right), \forall p \in \mathbb{N},
$$

i.e., $\left|\left\langle t_{p}, x_{0}^{*}-x_{m_{k}}^{*}\right\rangle\right| \geq \beta, \quad k=1,2, \ldots, p$. Choose a subsequence of $\left(t_{n}\right)$ (denoted again by $\left(t_{n}\right)$ ) such that there exists $\lim _{n}\left\langle t_{n}, x_{0}^{*}-x_{m_{k}}^{*}\right\rangle$, for any $k$. Then we get

$$
\begin{aligned}
\lim _{n} \lim _{k}\left\langle t_{n}, x_{m_{k}}^{*}\right\rangle & =\lim _{n}\left\langle t_{n}, x_{0}^{*}\right\rangle, \\
\left|\lim _{n}\left\langle t_{n}, x_{0}^{*}\right\rangle-\lim _{k} \lim _{n}\left\langle t_{n}, x_{m_{k}}^{*}\right\rangle\right| & =\lim _{k} \lim _{n}\left|\left\langle t_{n}, x_{0}^{*}-x_{m_{k}}^{*}\right\rangle\right| \geq \beta,
\end{aligned}
$$

So

$$
\left|\lim _{n} \lim _{k}\left\langle t_{n}, x_{m_{k}}^{*}\right\rangle-\lim _{k} \lim _{n}\left\langle t_{n}, x_{m_{k}}^{*}\right\rangle\right| \geq \beta
$$

As $\beta$ satisfies $0<\beta<\varepsilon$ and it is otherwise arbitrary, we get the conclusion. 
Proof of Theorem 2. The general case follows from Proposition 8 and Theorem 13. In order to prove the case when $\left(B_{X^{*}}, \omega^{*}\right)$ is angelic, the following modification of Proposition 8 is needed, together with the fact that, according to the proof of Theorem 13, if some sequence in the convex hull of a set "fails" the double limit condition against a sequence $\left(x_{m}^{*}\right)_{m \in \mathbb{N}}$ in the dual, the same is true for the set and a certain subsequence of $\left(x_{m}^{*}\right)_{m \in \mathbb{N}}$ :

Proposition 14 Let $M$ be a bounded set and let $\varepsilon>0$. Then

(i) If $M$ is $\varepsilon-W R K$ then $M \S \varepsilon \S\left(x_{n}^{*}\right)$, where $\left(x_{n}^{*}\right)$ is any $w^{*}-$ null sequence in $B_{X^{*}}$.

(ii) If $\left(B_{X^{*}}, \omega^{*}\right)$ is angelic and $M \S \varepsilon \S\left(x_{n}^{*}\right)$ for any $w^{*}-$ null sequence in $B_{X^{*}}$ then $M$ is $\varepsilon-W R K$.

Proof. $(i)$ follows directly from the proof of $(i)$ in Proposition 8. In order to prove $(i i)$, let

$$
x_{0}^{* *} \in \bar{M}^{\left(X^{* *}, \omega^{*}\right)},
$$

and let $d:=\operatorname{dist}\left(x_{0}^{* *}, X\right)$. There exists $x_{0}^{* * *} \in S_{X^{* * *}}$ such that

$$
\left\langle x_{0}^{* *}, x_{0}^{* * *}\right\rangle=d, \quad\left\langle x, x_{0}^{* * *}\right\rangle=0, \text { for all } x \in X .
$$

Fix $\delta>0$. Let

$$
C:=B_{X^{*}} \cap\left\{x^{* * *} \in X^{* * *} ;\left\langle x_{0}^{* *}, x^{* * *}\right\rangle \in(d-\delta, d+\delta)\right\} .
$$

Then

$$
x_{0}^{* * *} \in \bar{C}^{\left(X^{* * *}, \omega^{*}\right)}
$$

and then

$$
0 \in \bar{C}^{\left(X^{*}, \omega^{*}\right)} .
$$

As $\left(B_{X^{*}}, \omega^{*}\right)$ is angelic, there exists a sequence $\left(x_{m}^{*}\right)_{m \in \mathbb{N}}$ in $C$ which converges to 0 in $\left(X^{*}, \omega^{*}\right)$. By passing to a subsequence (denoted by the same symbol) we may and do assume that $\left(\left\langle x_{0}^{* *}, x_{m}^{*}\right\rangle\right)_{m \in \mathbb{N}}$ converges.

Let $\left(x_{n}\right)_{n \in \mathbb{N}}$ be a sequence in $M$ which converges to $x_{0}^{* *}$ in $X^{* *}$ on the set $\left\{x_{m}^{*} ; m \in \mathbb{N}\right\}$. Then

$$
\begin{aligned}
\lim _{n} \lim _{m}\left\langle x_{n}, x_{m}^{*}\right\rangle & =0, \quad \text { and } \\
\lim _{m} \lim _{n}\left\langle x_{n}, x_{m}^{*}\right\rangle & =\lim _{m}\left\langle x_{0}^{* *}, x_{m}^{*}\right\rangle \in[d-\delta, d+\delta] .
\end{aligned}
$$

As $\delta>0$ was arbitrary, we are done. 
To finish the proof of Theorem 2 it remains to show the case when $X^{*}$ does not contains a copy of $\ell^{1}$ : let $C:=\overline{\operatorname{conv}(M)}^{w^{*}}$, a $w^{*}$-compact convex subset of $X^{* *}$. It is well known (see, for example, [3, p. 215]) that

$$
C=\overline{\operatorname{conv}(\operatorname{Ext} C)}\|\cdot\|,
$$

where $E x t C$ denotes the set of extreme points of $C$. By Milman's Theorem (see, for example, $[11, \S 25.1 .7]), \operatorname{Ext} C \subset \bar{M}^{w^{*}}$. As

$$
\left\{x^{* *} \in X^{* *}: d\left(x^{* *}, X\right) \leq \varepsilon\right\}
$$

is $\|\cdot\|$-closed, where $d$ denotes the distance in the norm, this proves that $\overline{\operatorname{conv}(M)}$ is $\varepsilon-\mathrm{WRK}$.

\section{Applications}

In [5] a characterization of subspaces of weakly compact generated Banach spaces was provided in terms of countable coverings of the closed unit ball by absolutely convex subsets $\left(M_{n, p}\right)_{n, p \in \mathbb{N}}$. Using Theorem 2 , the convexity requirement on those sets can be removed, and the following is true:

Theorem 15 ([5]) A Banach space $X$ is a subspace of a weakly compact generated Banach space if and only if it admits a family $\left\{M_{n, p} ; n, p \in \mathbb{N}\right\}$ of subsets of $B_{X}$ such that

$$
\bigcup_{n=1}^{\infty} M_{n, p}=B_{X}
$$

for every $p \in \mathbb{N}$, and

$$
{\overline{M_{n, p}}}^{w^{*}} \subset X+\frac{1}{p} B_{X^{* *}}
$$

for every $n, p \in \mathbb{N}$.

Acknowledgements. The authors would like to thank Y. Benyamini for informing us that a separable version of the result had been proved some time ago by H. P. Rosenthal (unpublished). We also thank H. P. Rosenthal for a correspondence concerning his result and G. Godefroy for several enlightening discussions on this subject. Finally, we thank the referee for many suggestions which helped to improve both the form and the results of this paper.

Added in Proof: The problem mentioned in 3 has been solved recently in the negative by A. Suárez-Granero in a forthcoming paper (see [8]). Assuming $\mathrm{CH}$, he provides an example of a space where the best constant in the quantitative Krein's Theorem is 2. Thus the positive result in our paper is in fact optimal. See also [9] for another example without $\mathrm{CH}$. 


\section{References}

[1] Bellenot, S. F., Haydon, R. and Odell, E.: Quasi-reflexive and tree spaces constructed in the spirit of R. C. James. In Banach space theory (Iowa City, IA, 1987), 19-43. Contemp. Math. 85. Amer. Math. Soc., Providence, RI, 1989.

[2] Deville, R., Godefroy, G. And Zizler, V.: Smoothness and Renormings in Banach Spaces. Pitman Monographs and Surveys in Pure and Applied Mathematics 64. Longman Scientific \& Technical, Harlow, 1993.

[3] Diestel, J.: Sequences and Series in Banach Spaces. Graduate Texts in Mathematics 92. Springer-Verlag, New York, 1984.

[4] Eberlein, W. F.: Weak compactness in Banach spaces, I. Proc. Natl. Acad. Sci. USA 33 (1947), 51-53.

[5] Fabian, M., Montesinos, V. And Zizler, V.: A characterization of subspaces of weakly compactly generated Banach spaces. J. London Math. Soc. (2) 69 (2004), no. 2, 457-464.

[6] Fabian, M., Habala, P., Hájek, P., Pelant, J., Montesinos, V. And Zizler, V.: Functional Analysis and Infinite Dimensional Topology. CMS Books in Mathematics 8. Springer Verlag, New York, 2001.

[7] Godefroy, G. and Kalton, N.; The ball topology and its applications. In Banach space theory (Iowa City, IA, 1987), 195-237. Contemp. Math. 85. Amer. Math. Soc., Providence, RI, 1989.

[8] Granero, A.S.: An extension of the Krein-S̆mulyan Theorem in Banach spaces. To appear in Rev. Mat. Iberoamericana.

[9] Granero, A.S., Háyek, P. and Montesinos, V.: Convexity and $w^{*}$-compactness in Banach spaces. Math. Ann. 328 (2004), 625-631.

[10] Grothendieck, A.: Critères de compacité dans les espaces fonctionnels généraux. Amer. J. Math. 74 (1952), 168-186.

[11] Köтнe, G.: Topological Vector Spaces I. Die Grundlehren der mathematischen Wissenschaften 159. Springer-Verlag, New York, 1969.

[12] PтÁk, V.: A combinatorial lemma on the existence of convex means and its applications to weak compactness. In 1963 Proc. Sympos. Pure Math. vol. VIII, 437-450. Amer. Math. Soc. Providence, RI, 1963.

[13] Rosenthal, H. P.: The heredity problem for weakly compactly generated Banach spaces. Compositio Math. 28 (1974), 83-111.

Recibido: 5 de noviembre de 2002.

Revisado: 17 de enero de 2003. 
M. Fabian

Mathematical Institute of the Czech Academy of Sciences

Žitná 25

11567, Prague 1, Czech Republic

fabian@math.cas.cz

P. Hájek

Mathematical Institute of the Czech Academy of Sciences

Žitná 25

11567, Prague 1, Czech Republic

hajek@matsrv.math.cas.cz

V. Montesinos

Departamento de Matemática Aplicada

ETSI Telecomunicación, Universidad Politécnica de Valencia

C/Vera, s/n.

46071-Valencia, Spain

vmontesinos@mat.upv.es

V. Zizler

Department of Mathematical Sciences

University of Alberta

632 Central Academic Building

Edmonton, Alberta T6G 2G1, Canada

vzizler@math.ualberta.ca

M. Fabian is supported by grants AV 1019003 and GAČR 201/01/1198. P. Hájek is supported by grants A1 019205, AV 1019003, GAČR 201/01/1198 and by a grant from the Universidad Politécnica de Valencia. This author acknowledges the hospitality and working conditions provided by this University. V. Montesinos is supported in part by Project PB96-0758(Spain), Project BFM2002-01423 and by the Universidad Politécnica de Valencia. V. Zizler is supported by NSERC 7926-02 (Canada). 